\title{
Vascular Accessibility of Endothelial Targeted Ferritin Nanoparticles
}

Makan Khoshnejad, Vladimir V. Shuvaev, Katherine W. Pulsipher, Chuanyun Dai, Elizabeth D. Hood, Evguenia Arguiri, Melpo Christofidou-Solomidou, Ivan J. Dmochowski, Colin F. Greineder, and Vladimir R. Muzykantov

\section{List of Contents for Supporting Information.}

Figure S1. Analysis of conjugate stoichiometry

Figure S2. HPLC absorbance traces

Figure S3. Biodistribution of anti-PECAMscFv/Ft

Table S1. Raw data for biodistribution studies

Table S2. Antibody clone names Figures S1-3, Tables S1-2. 


\section{Supporting Information}

a)

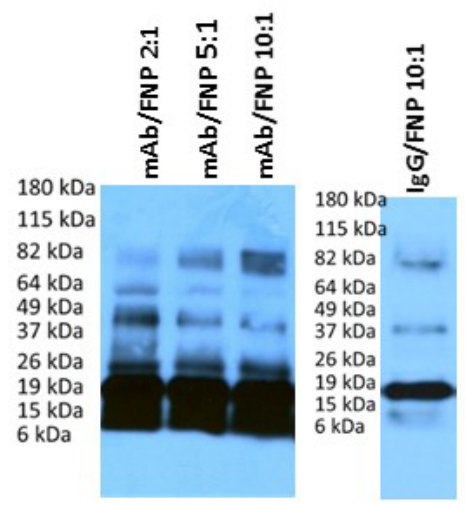

d)

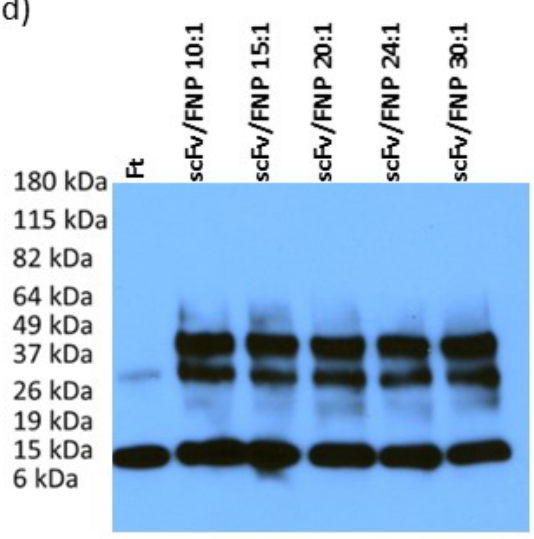

b)

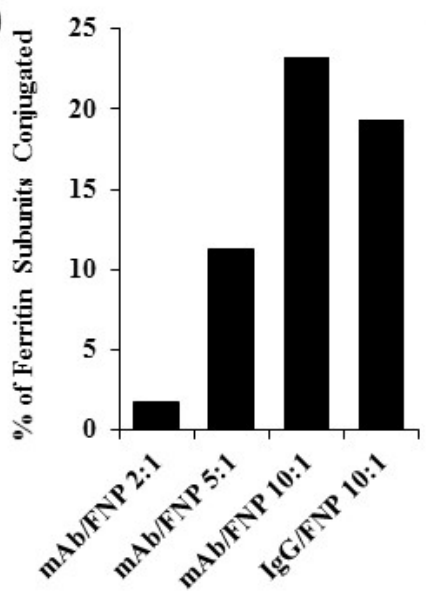

e)

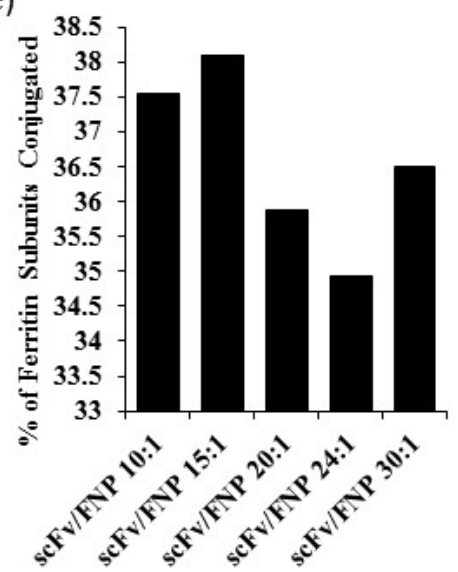

c)

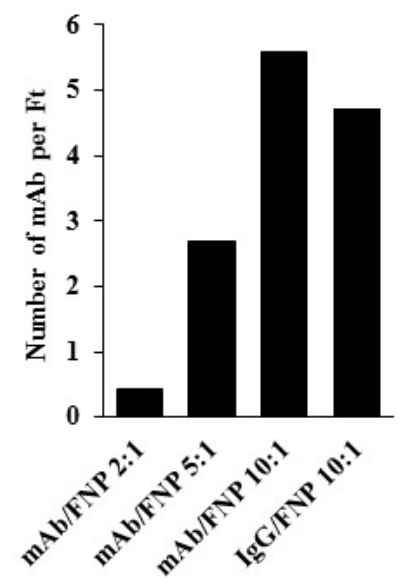

f)

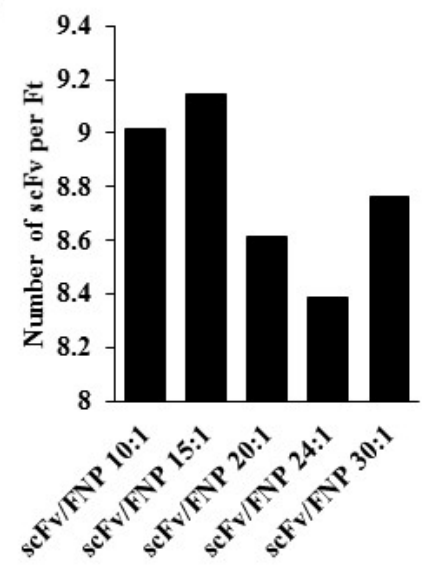

Figure S1. Analysis of conjugate stoichiometry. Western blot of a) anti-ICAM Ab/FNP, an unrelated IgG/FNP, and d) anti-PECAM scFv/FNP conjugates at different molar ratios stained with anti-horse ferritin antibody. Quantitative western blot analysis of ferritin conjugated to b) anti-ICAM Ab and e) anti-PECAM scFv. Percent conjugates is the densitometric ratio of conjugates band to total. The estimated number of c) whole antibodies and f) scFv antibody fragments conjugated is calculated by the percent antibody conjugated multiplied by 24 (subunits of ferritin). 

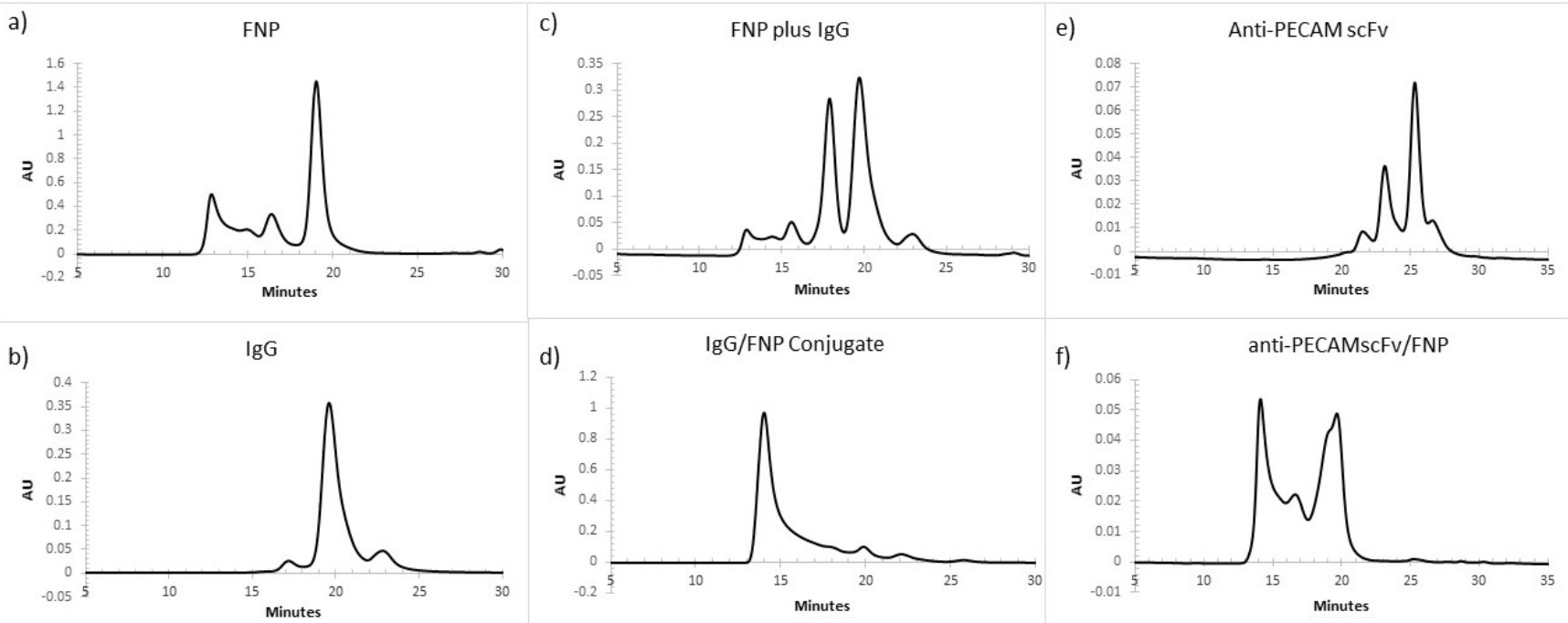

Figure S2. HPLC absorbance traces for a) horse spleen FNP, b) IgG, c) mixture of FNP plus IgG, d) conjugated IgG/FNP, e) anti-PECAM scFv, and f) anti-PECAM scFv/FNP conjugate.

a)

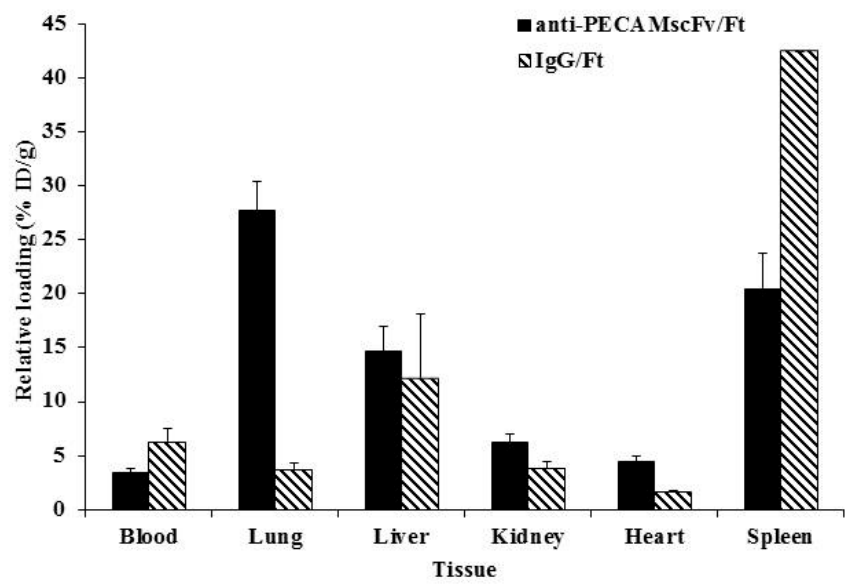

b)

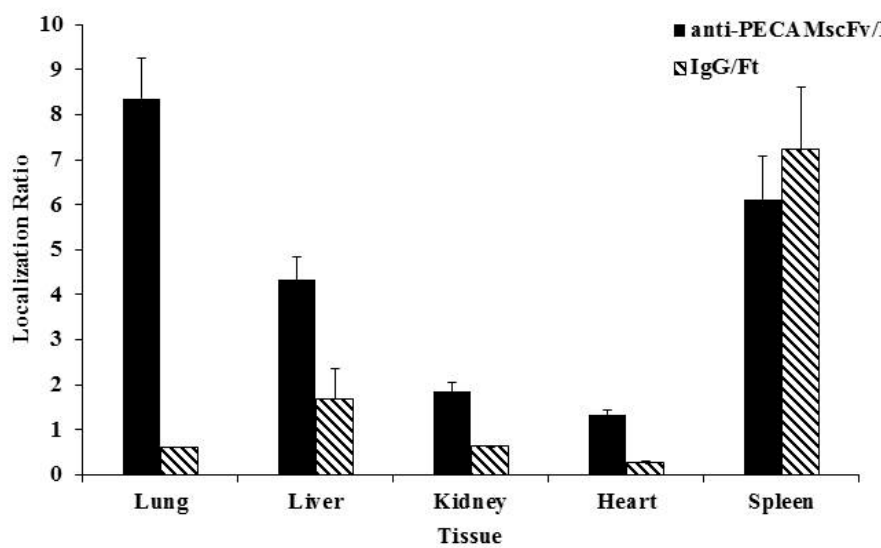

Figure S3. Targeting of FNPs to PECAM-1. a) Biodistribution of ${ }^{125}$ I-labeled anti-PECAM scFv and IgG FNPs in mice at 30 min. Tissue uptake is indicated as mean \pm SEM ( $n=3) . \quad b)$ Localization ratio of selected organs. Significant differences determined by t-test with Bonferroni correction to account for multiple comparisons. 


\begin{tabular}{|l|c|c|c|c|c|c|c|}
\hline Group & & Blood & Lung & Liver & Kidney & Heart & Spleen \\
\hline anti-ICAM Ab/FNP & mean & 4.084 & 160.9 & 23.43 & 7.428 & 3.505 & 28.54 \\
\hline IgG/FNP & SEM & 1.761 & 6.468 & 1.048 & 0.690 & 0.333 & 4.684 \\
\hline & mean & 5.265 & 14.54 & 49.37 & 3.109 & 0.834 & 39.42 \\
\hline
\end{tabular}

\begin{tabular}{|l|c|c|c|c|c|c|c|}
\hline Group & & & & & & & \\
\hline anti-PECAM Ab/FNP & mean & 3.194 & 85.35 & 20.57 & 8.270 & 4.685 & 23.98 \\
\hline IgG/FNP & SEM & 0.049 & 2.119 & 1.301 & 0.198 & 0.217 & 0.856 \\
\hline & Mean & 4.710 & 11.91 & 43.39 & 3.352 & 1.095 & 51.77 \\
\hline & SEM & 0.285 & 1.181 & 2.887 & 0.177 & 0.110 & 5.717 \\
\hline
\end{tabular}

Table S1. Raw data for biodistribution studies showing the mean \%ID/g for each organ and their standard error mean (SEM).

\begin{tabular}{|l|l|}
\hline Antibody & Clone name \\
\hline anti-mICAM Ab & YN1 mAb \\
\hline anti-mPECAM scFv & $390 \mathrm{scFv}$ \\
\hline Anti-mPECAM Ab & Mec13.3 mAb \\
\hline anti-hICAM Ab & R6.5 mAb \\
\hline anti-hPECAM Ab & Ab62 mAb \\
\hline
\end{tabular}

Table S2. Clone names corresponding to the antibodies used in this study. 